\title{
The Effectiveness and Applicability of Compensatory Cognitive Training for Japanese Patients with Schizophrenia: A Pilot Study
}

\author{
Sadao Otsuka, ${ }^{1}$ Mie Matsui, ${ }^{1}$ Takatoshi Hoshino, ${ }^{1}$ Kayoko Miura, ${ }^{1}$ \\ Yuko Higuchi, ${ }^{2}$ and Michio Suzuki ${ }^{2}$ \\ ${ }^{1}$ Department of Psychology, Graduate School of Medicine and Pharmaceutical Sciences, University of Toyama, \\ Toyama 930-0194, Japan \\ ${ }^{2}$ Department of Neuropsychiatry, Graduate School of Medicine and Pharmaceutical Sciences, University of Toyama, \\ Toyama 930-0194, Japan \\ Correspondence should be addressed to Mie Matsui; mmatsui@las.u-toyama.ac.jp
}

Received 13 March 2015; Revised 14 June 2015; Accepted 16 June 2015

Academic Editor: Raphael J. Braga

Copyright (C) 2015 Sadao Otsuka et al. This is an open access article distributed under the Creative Commons Attribution License, which permits unrestricted use, distribution, and reproduction in any medium, provided the original work is properly cited.

\begin{abstract}
Although cognitive remediation or training for schizophrenia has been developed, few studies on the subject have focused on Japanese patients. The aim of the present study was to examine the effectiveness and applicability of compensatory cognitive training (CCT) in Japanese patients with schizophrenia. Twenty-six participants diagnosed with schizophrenia were assigned to either the CCT plus treatment as usual group $(n=13)$ or the treatment as usual alone group $(n=13)$. CCT is a 12-session, manualized, groupbased training that coaches compensatory strategies in four cognitive domains (prospective memory, attention, verbal memory, and executive functions). Cognitive, functional, and clinical symptom measures were implemented at baseline, after treatment, and at 3-month follow-up. Mixed design analyses of variance with group and time for each measure demonstrated that effects of CCT on verbal memory, processing speed, and social functioning at postintervention were significant, and the effects on processing speed were maintained at follow-up. Our study suggests that CCT has beneficial effects on cognitive performance, improving functional outcomes in Japanese patients with schizophrenia. Additionally, the high degrees of attendance rates and level of satisfaction rated by the CCT participants ensure the applicability of this methodology to this population.
\end{abstract}

\section{Introduction}

Cognitive impairment is broadly considered as one of the core components of schizophrenia, along with well-known psychotic symptoms such as positive and negative symptoms [1]. Previous studies have reported that the severity of cognitive impairments in individuals with schizophrenia is related to difficulties in social participation and could predict later functional outcomes including employment and independence in everyday living, though the explained variance in functional outcomes was modest [2-6]. While the progress of antipsychotic medications can serve to reduce positive symptoms dramatically, improvements in everyday functioning and the promotion of social participation remain as issues to be solved. Consequently, there is strong interest in advancing interventions aimed at improving cognitive performance, leading to the enhancement of everyday and social functioning in individuals with schizophrenia.

Cognitive impairments in schizophrenia are evident in a wide range of domains, including attention, memory, and executive functions [7-10]. The psychotic symptoms in schizophrenia are manifested more commonly in adolescents and young adults and require lifelong treatment to stabilize. In contrast, cognitive impairments are known to appear before the symptomatic manifestation of the illness [11-14] and are relatively stable over time after the onset of the illness [15]. Importantly, performance on the cognitive tasks shows the weakest or no relationship with positive symptoms, 
though a significant relationship with negative symptoms is reported [16-20]. These findings support the idea that cognitive impairments in schizophrenia are relatively independent of psychotic symptoms, or at least of positive symptoms [1].

The treatment of schizophrenia predominantly relies on pharmaceutical drugs. Thus, there are a number of studies examining the improvement effects of antipsychotic drugs on cognitive impairments in schizophrenia. Specifically, studies report that significant effects of antipsychotic drugs on cognitive functions were observed when comparing cognitive performance before and after treatment, but no differences were observed when comparing the effects of atypical and typical drugs on cognitive performance $[21,22]$. In addition, the magnitude of cognitive improvements associated with atypical drugs is relatively small and in fact is similar to practice effects in healthy controls [23]. Thus, the effects of antipsychotic medications on cognitive functions are far from satisfactory, and little is known about the improvement effects of antipsychotic drugs on everyday and social functioning in patients with schizophrenia.

Against this background, cognitive remediation or training for schizophrenia has been developed as a psychosocial therapeutic approach to directly address cognitive impairments. Cognitive remediation is defined as "a behavioral training-based intervention that aims to improve cognitive processes (attention, memory, executive function, social cognition, or metacognition) with the goal of durability and generalization" [24]. The effectiveness of cognitive remediation has been repeatedly demonstrated through randomized controlled trials and meta-analyses. For example, Wykes et al. [24] conducted a meta-analysis and showed that the improvement effect of cognitive remediation on global cognition was medium (Cohen's $d=0.45$, group comparison after treatment). This study also showed that the effects of cognitive remediation on global cognition were durable after the completion of the intervention $(d=0.43$, a comparison between pretreatment and follow-up evaluations within the cognitive remediation group). The effect sizes reported in their meta-analytic study on the respective cognitive domains were small to medium ( $d=0.25$ on attention, $d=0.26$ on processing speed, $d=0.35$ on verbal working memory, $d=0.41$ on verbal learning/memory, $d=0.57$ on reasoning/problem solving, and $d=0.65$ on social cognition). The effect sizes did not differ according to the type of intervention approach, duration of treatment, or use of computers. In addition, the authors found that cognitive remediation had a small-to-medium effect (0.42) on functional outcomes. Regarding the effectiveness of cognitive remediation on psychosocial functioning, McGurk et al. [25] conducted a meta-analysis and demonstrated that cognitive remediation programs that used strategy coaching in addition to the drilland-practice method had greater effects $(d=0.62)$ when compared to programs using the drill-and-practice method alone $(d=0.24)$. Wykes et al. [24] also confirmed this finding.

These findings suggest that we could expect to obtain equivalent effects of cognitive remediation in Japanese individuals with schizophrenia. However, to date, only four studies have reported the effectiveness and validity of cognitive remediation for schizophrenia in Japan, all without referring to strategy coaching [26-29]. Therefore, in this study, we focused on a compensatory cognitive training (CCT) that includes strategy coaching developed by Twamley and colleagues, the effectiveness of which has been sufficiently demonstrated in the US $[30,31]$. Although the term "strategy coaching" is not well defined, it commonly includes instructions on using cognitive or behavioral strategies to cope with cognitive activities effectively, to avoid overloading one's cognitive capability. The characteristic feature of CCT is that the approach focuses on compensatory strategies that the participants can use on their own in real-world conditions. Compensatory approach, in contrast to restorative approaches such as utilizing repetitive drill practices [32, 33 ], is intended for the participants to acquire new ways to apply their capability in order to compensate for the cognitive impairments and interact with the world suitably. The compensatory strategies coached in the CCT intervention include not only the use of external or environmental aids (e.g., writing down the things to do later) but also internal or psychological attempts (using categorization or visual imagery to retain information). Specifically, acquisition of these internal strategies would improve cognitive performance evaluated using neuropsychological tests, without the use of any external tools during a test circumstance and any drill in the CCT intervention, as reported in previous studies $[30,31]$. We translated the therapist's manual and the participant's workbook of CCT $[30,31]$ into Japanese and partially modified them while considering the cultural backgrounds of the Japanese participants. The following modifications were made. First, changes to the scenarios used for role-play training were made and some scenarios were added. Second, the commonly used "Goroawase" method was added, in which the participants create a meaningful sentence by utilizing the phonological aspects of a series of digits (like a wordplay), when memorizing digits such as a phone number or a year in which a historical event occurred. As a preliminary step of effect research on CCT for Japanese patients with schizophrenia, we need to confirm that the Japanese version has not lost the advantages of CCT during the translation process and after the modifications mentioned above.

The aim of this pilot study was to examine the effectiveness and applicability of CCT for Japanese patients with schizophrenia. We predicted that the application of CCT in Japanese patients with schizophrenia would benefit cognitive and functional outcomes, as was noted in previous studies $[30,31]$. The improvement effects of CCT on cognitive performance combined with antipsychotic medication would be greater than the effectiveness of antipsychotic medication alone. In addition, CCT should positively operate upon the everyday and social functioning of patients with improvements in cognitive performance. We expected these improvement effects to be durable at follow-up evaluations implemented several months after the completion of the CCT intervention. We also expected that the CCT intervention would be feasible with Japanese individuals with schizophrenia, as reflected in high attendance rates and high satisfaction reported by participants. 


\section{Method}

2.1. Protocol. The study protocol is presented in Figure 1. Our trial compared CCT plus treatment as usual (CCT group) with treatment as usual alone (TAU group). The CCT intervention was delivered over 12 weeks in weekly 2-hour group sessions for the CCT group. Cognitive measures (neuropsychological tests), functional measures (questionnaires asking participants and their families about everyday and social functioning), and clinical symptom measures were implemented at preintervention (baseline, T1), immediately following the completion of CCT (postintervention, T2), and three months following the completion of CCT (follow-up, T3), to evaluate the efficacy of CCT. It was explained to the participants in both groups that the tests would assess their current status, and they were encouraged to perform well to the extent that they could at the three time points. Clinical symptoms were assessed by the Scale for the Assessment of Positive Symptoms [34] and the Scale for the Assessment of Negative Symptoms [35]. The study was approved by the Committee on Medical Ethics of the University of Toyama, and all participants provided written informed consent prior to participating in the study.

2.2. Participants. Participants included inpatients and outpatients recruited from the same hospital. All participants were diagnosed with schizophrenia or schizoaffective disorder according to DSM-IV-TR criteria [36]. The participants were aged 18 years or older and showed evidence of cognitive difficulties (i.e., scored more than 1 SD below average on at least one of the neuropsychological tests at baseline). They maintained a stable dose of medication for at least 1 month prior to inclusion in the study. The following exclusion criteria were applied: neurological illness, traumatic head injury, current substance or alcohol abuse, and an estimate of premorbid IQ > 90 as assessed by the Japanese Adult Reading Test (JART) [37]. The demographic and clinical characteristics of the participants are presented in Table 1.

Twenty-six participants were enrolled in the study. We used posters and brochures to recruit patients for the study. All participants were recommended for the study by their attending psychiatrist. Thirteen participants were assigned to the CCT group and the other 13 to the TAU group. The CCT and TAU groups did not differ statistically on age, gender, years of education, duration of illness, age at onset, antipsychotic dose (risperidone equivalents) [38], symptom severity, or any of the neuropsychological tests at the beginning of the CCT intervention (i.e., baseline). More than half the participants were living with their families (65\%), about onethird were in the hospital (31\%), and only one participant was living alone (4\%). Some participants worked part-time (12\%) and the others were unemployed (88\%). In the CCT group, 12 participants completed the postintervention evaluations and 11 completed the follow-up evaluations; in the TAU group, 11 completed the postintervention evaluations and 8 completed the follow-up evaluations.

2.3. CCT Intervention. The CCT intervention delivered in the study was a 12-session, manualized, group-based training

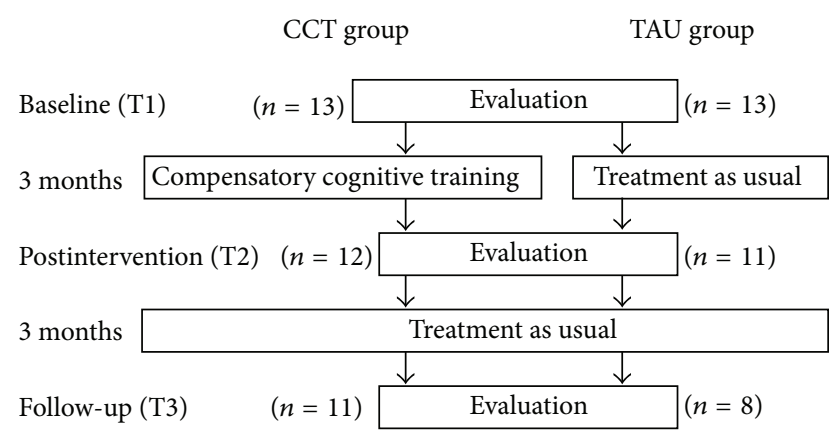

FIGURE 1: Flowchart of the study design. CCT: compensatory cognitive training. TAU: treatment as usual. $n$ : number of patients who finished evaluations at each time point. The CCT group received the CCT intervention plus treatment as usual for 3 months after baseline evaluations. The TAU group received treatment as usual for 3 months after baseline evaluations. After that, both groups underwent postintervention assessment and follow-up evaluations after 3 months of treatment as usual.

focusing on the 4 cognitive domains presented in Table 2 . The CCT groups consisted of fewer than 6 participants and more than 2 therapists who were certified clinical psychologists and a doctoral-level clinical neuropsychologist. Before starting the study, we role-played all 12 sessions with members of our laboratory as staff training in order to enhance the fidelity of the treatment procedure and refine the Japanese manual.

In this approach, homework assignments are important. Homework is assigned at the end of each session and enables participants to identify situations in which they would use a given strategy. In addition, participants are provided with an opportunity to troubleshoot any difficulties concerning the homework at the beginning of each session. These procedures encourage participants to develop learned strategies into new habits in their real-world circumstances. The learning of habits has been shown to be intact in individuals with schizophrenia [39, 40], and habits are particularly resistant to forgetting [41]. Thus, the effects of the compensatory approach based on habit learning are expected to endure for a longer period of time and be generalized to everyday living and social activities.

CCT covers prospective memory, attention/vigilance, verbal learning/memory, and executive functions, in that order. These four cognitive domains were selected based on their degree of impairment in schizophrenia spectrum disorders, relevance for psychosocial functioning, and potential modifiability $[4,42,43]$. In addition, CCT has the following features: no use of computer and being delivered anywhere, interactive, game-like format as much as possible to maintain the interest and attention of participants, and a workbook whereby participants could review the things they had worked on anywhere and anytime. A short break between the first and second hours of each session is given to reduce fatigue (see $[30,31]$ for more details).

\subsection{Measures}

2.4.1. Cognitive Measures. The neuropsychological tests were implemented as cognitive measures in the following cognitive 
TABLE 1: Baseline group comparison of participants $(n=26)$.

\begin{tabular}{|c|c|c|c|c|c|}
\hline & $\begin{array}{c}\text { CCT }(n=13) \\
\text { Mean }(\mathrm{SD})\end{array}$ & $\begin{array}{c}\text { TAU }(n=13) \\
\text { Mean }(\mathrm{SD})\end{array}$ & $t$ or $\chi^{2}$ & $p$ value & $d$ \\
\hline \multicolumn{6}{|l|}{ Demographics } \\
\hline Age (years) & $34.6(10.2)$ & $39.5(8.9)$ & 1.31 & 0.20 & 0.51 \\
\hline Gender (\% male) & $61.5 \%$ & $46.2 \%$ & 0.62 & 0.43 & \\
\hline Education (years) & $14.7(1.5)$ & $14.0(1.8)$ & 1.06 & 0.30 & 0.42 \\
\hline \multicolumn{6}{|l|}{ Illness burden } \\
\hline Illness duration (years) & $11.8(6.8)$ & $16.5(9.4)$ & 1.61 & 0.16 & 0.57 \\
\hline Age at onset (years) & $22.8(6.6)$ & $22.9(7.5)$ & 0.01 & 0.94 & 0.02 \\
\hline Antipsychotic dose (mg/day, RPD equivalent) & $5.5(3.6)$ & $5.7(3.6)$ & 0.11 & 0.91 & 0.06 \\
\hline SAPS positive symptoms & $19.5(14.3)$ & $29.4(21.8)$ & 1.36 & 0.19 & 0.54 \\
\hline SANS negative symptoms & $43.3(17.4)$ & $43.5(20.0)$ & 0.12 & 0.98 & 0.01 \\
\hline \multicolumn{6}{|l|}{ Cognitive functions } \\
\hline \multicolumn{6}{|l|}{ Verbal memory } \\
\hline JVLT total score & $29.5(6.9)$ & $28.1(7.7)$ & 0.74 & 0.62 & 0.19 \\
\hline RBMT immediate recall & $13.2(4.9)$ & $13.0(4.7)$ & 0.10 & 0.92 & 0.04 \\
\hline \multicolumn{6}{|l|}{ Processing speed } \\
\hline Digit symbol raw score & $70.5(16.0)$ & $76.2(21.2)$ & 0.77 & 0.45 & 0.30 \\
\hline TMT-A time & $40.0(13.2)$ & $35.9(11.4)$ & 0.84 & 0.41 & 0.33 \\
\hline \multicolumn{6}{|l|}{ Executive functioning } \\
\hline WCST completed category & $5.5(1.7)$ & $5.2(2.1)$ & 0.42 & 0.68 & 0.16 \\
\hline TMT-B time & $77.6(34.7)$ & $75.8(24.3)$ & 0.15 & 0.88 & 0.06 \\
\hline
\end{tabular}

RPD $=$ risperidone, SAPS $=$ Scale for Positive Symptoms, SANS $=$ Scale for Negative Symptoms, JVLT $=$ Japanese Verbal Learning Test, RBMT $=$ Rivermead Behavioral Memory Test, TMT-A = Trail Making Test-A, WCST = Wisconsin Card Sorting Test, and TMT-B = Trail Making Test-B.

TABLE 2: Domains and strategies included in compensatory cognitive training [31].

\begin{tabular}{|c|c|c|}
\hline $\begin{array}{l}\text { Targeted } \\
\text { domain }\end{array}$ & Importance for everyday functioning & Compensatory strategies \\
\hline $\begin{array}{l}\text { Prospective } \\
\text { memory }\end{array}$ & $\begin{array}{l}\text { (i) Remembering to go to work or school } \\
\text { (ii) Remembering to take medications } \\
\text { (iii) Remembering to turn in school assignments } \\
\text { (iv) Remembering to do assigned tasks at work in } \\
\text { response to cues }\end{array}$ & $\begin{array}{l}\text { (1) Daily calendar use } \\
\text { (2) To-do lists and prioritizing tasks } \\
\text { (3) Linking tasks by using reminders or using routines } \\
\text { to automate tasks } \\
\text { (4) Automatic place }\end{array}$ \\
\hline $\begin{array}{l}\text { Attention and } \\
\text { vigilance }\end{array}$ & $\begin{array}{l}\text { (i) Paying attention to communications from } \\
\text { supervisors and coworkers } \\
\text { (ii) Maintaining attention in class or while studying } \\
\text { (iii) Maintaining attention for work tasks or household } \\
\text { projects without getting distracted }\end{array}$ & $\begin{array}{l}\text { (1) Conversational vigilance skills (reduce distractions, } \\
\text { eye contact, paraphrasing, and asking questions) } \\
\text { (2) Task vigilance skills (use self-talk during tasks to } \\
\text { maintain focus, taking breaks to refocus) }\end{array}$ \\
\hline $\begin{array}{l}\text { Learning and } \\
\text { memory }\end{array}$ & $\begin{array}{l}\text { (i) Learning and remembering work tasks } \\
\text { (ii) Learning novel information in school or vocational } \\
\text { training } \\
\text { (iii) Learning and remembering names of supervisors } \\
\text { and coworkers }\end{array}$ & $\begin{array}{l}\text { (1) Encoding strategies (taking notes, paraphrasing, } \\
\text { association, chunking, categorizing, acronyms, rhymes, } \\
\text { visual imagery, and overlearning) } \\
\text { (2) Retrieval strategies } \\
\text { (3) Name-learning strategies }\end{array}$ \\
\hline $\begin{array}{l}\text { Executive } \\
\text { functioning }\end{array}$ & $\begin{array}{l}\text { (i) Problem solving and coping with unexpected } \\
\text { situations on the job or in vocational training, or at } \\
\text { home } \\
\text { (ii) Being able to balance demands of work/school with } \\
\text { home/family needs } \\
\text { (iii) Thinking flexibly and self-monitoring performance } \\
\text { at work }\end{array}$ & $\begin{array}{l}\text { (1) 6-step problem solving method (define problem, } \\
\text { brainstorm solutions, evaluate solutions systematically, } \\
\text { select a solution, try it, and evaluate how it worked) } \\
\text { (2) Self-talk while solving problems } \\
\text { (3) Hypothesis testing } \\
\text { (4) Self-monitoring }\end{array}$ \\
\hline
\end{tabular}


domains. All measures were administered by experienced psychologists trained in standardized testing procedures.

(1) Verbal learning/memory: the Japanese Verbal Learning Test (JVLT) [44, 45] based on Gold et al. [46], in which scores are given for the number of words recalled immediately $(0-48)$ and after a delay $(0-16$, see $[44,45]$ for more details), and the story subtest of the Japanese version of the Rivermead Behavioral Memory Test (RBMT) $[47,48]$, where scores are given for the number of sentences recalled immediately (0$25)$ and after a delay (0-25).

(2) Processing speed: digit symbols subtest of the Japanese version of the Wechsler Adult Intelligence Scale, third edition (WAIS-III) $[49,50]$, in which scores (0-133) are given for the number of symbols copied within $120 \mathrm{~s}$, and the Trail Making Test part A (TMT-A) [51], in which the time taken to complete the test was scored.

(3) Executive function: Wisconsin Card Sorting Test (WCST) [52, 53], where the number of categories completed was scored (0-6), and the Japanese version of the Trail Making Test part B (TMT-B) $[10,51]$, in which the time taken to complete the task was scored.

These cognitive measures, including some measures which were different from those used in previous studies $[30,31]$, were selected according to the content in the training (e.g., the RBMT story test for conversational attention, processing speed tests for task attention) and the possibility of improved performance in the tasks by applying the coached strategies. Prospective memory performance could be inferred from the attendance rate of the participants to some extent, although there was no standardized test with which to measure this domain in Japanese.

2.4.2. Functional Measures. The Social Functioning Scale Japanese version (SFS-J) $[54,55]$ was implemented as functional measures. SFS-J evaluates the functioning in everyday and social living, reported by participants and their families (score range: $0-226$ ).

2.5. Data Analyses. The statistical analyses were administered based on the intent-to-treat principle and therefore included all available data from the 26 participants completing the baseline evaluations regardless of whether they adhered to treatment (see Table 1). In order to compare the results of each measure on cognitive functions, everyday/social functioning, and clinical symptoms at three time points (baseline, T1; postintervention, T2; follow-up, T3), we performed twoway mixed factor analyses of variance (ANOVAs) with group (CCT versus TAU) as a between-subject factor and time points ( $\mathrm{T} 1$ versus $\mathrm{T} 2$ versus $\mathrm{T} 3$ ) as a within-subject factor. In these analyses, we adjusted the significance level (alpha) for the ANOVAs with Bonferroni correction. In the study, we focused on group-by-time interactions because the CCT intervention was considered effective when the improvement effects in the CCT group were beyond the effects of medication or natural course of time seen in the TAU group. Furthermore, we performed follow-up $t$-tests with Bonferroni correction (T2 versus T1, T3 versus T1) to examine changes from the baseline of each measure when the significant interactions were detected. In addition, we calculated Cohen's $d$ [56] using the change scores from baseline (T2-T1, T3-T1) of each measure to evaluate the magnitude of improvement effects with or without the CCT intervention.

\section{Results}

Table 3 presents the results of the mixed ANOVAs in terms of group-by-time interactions for all measures. The patterns of the significant interaction are seen in Figure 2. Two of the 11 participants in the CCT group who completed follow-up evaluations did not answer the questionnaires, so we analyzed data from 9 participants in the CCT group and 8 in the TAU group regarding functional measures.

3.1. Effects on Cognitive Functions. Regarding verbal memory, significant group-by-time interactions were found for JVLT total recall $\left(F=5.25, p=0.018\right.$, and $\left.\eta_{p}{ }^{2}=0.479\right)$. A series of Bonferroni-corrected paired comparisons revealed that the CCT group showed significant improvements in JVLT total recall at postintervention compared to baseline $(t=2.80$, $p=0.027$, and $d=1.26)$. In contrast, the TAU group did not show any changes from baseline on this measure $(t=1.21$, $p=0.839$, and $d=-0.47)$. Additionally, a similar trend was found on RBMT story immediate recall. The interaction was close to significance $\left(F=3.65, p=0.049\right.$, and $\eta_{p}{ }^{2}=$ 0.313 ), and paired comparisons indicated that the CCT group but not the TAU group showed significant improvements from baseline at postintervention $(t=3.28, p=0.021$, and $d=0.81$, Bonferroni-corrected). As for the follow-up period, there were no significant differences from baseline on all measures of verbal memory $(t s \leq 2.47, p s \geq 0.080$, and $d s \leq 0.61)$. That is, the improvement effects seen in the CCT group at postintervention were not maintained several months after the completion of CCT.

As shown in Table 3, significant interactions were found in processing speeds for both WAIS-III digit symbol and TMT-A ( $F s \geq 5.09, p s \leq 0.020$, and $\left.\eta_{p}{ }^{2} \geq 0.389\right)$. A series of paired comparisons indicated that the CCT group showed significant improvements from baseline on both digit symbol $(t=6.09, p=0.002$, and $d=0.66)$ and TMT-A $(t=5.36$, $p=0.000$, and $d=1.01$ ) at the postintervention period. Furthermore, the CCT group performed better at follow-up on both digit symbol $(t=3.66, p=0.002$, and $d=0.57)$ and TMT-A $(t=2.66, p=0.030$, and $d=0.59)$ compared to baseline, suggesting that the improvements seen in the CCT group on these cognitive measures were maintained for several months. The TAU group did not show significant changes on these measures at postintervention or follow-up compared to baseline ( $t \leq \leq 2.25, p s \geq 0.214$, and $d \leq 0.70$ ).

Regarding executive function, no significant interactions were found on WCST or TMT-B, as shown in Table 3 (Fs $\leq$ 1.12 , $p s \geq 0.350$, and $\eta_{p}^{2} \leq 0.123$ ). 


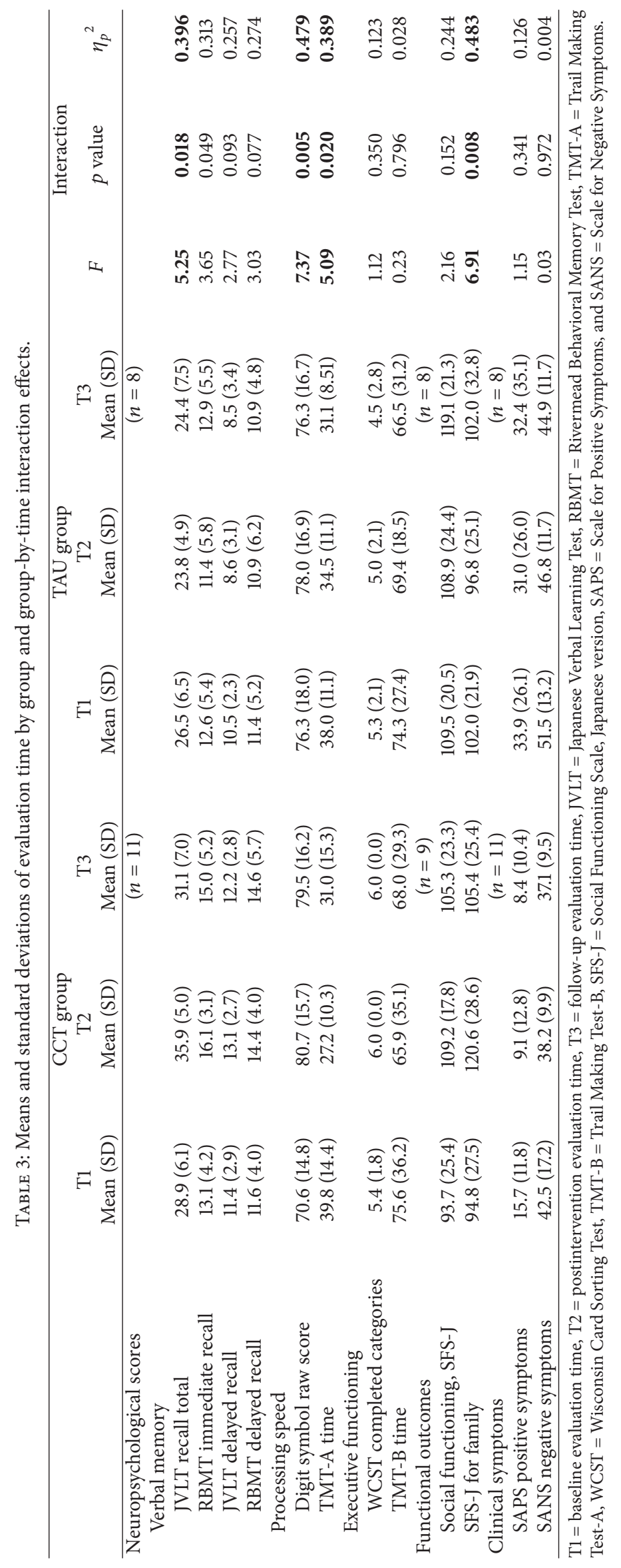




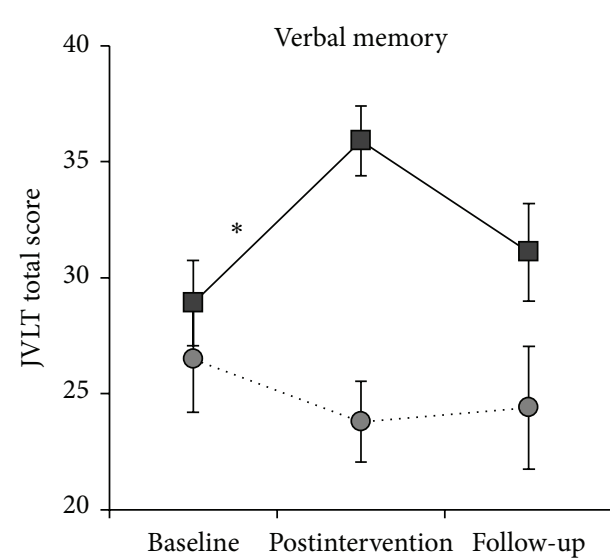

(a)

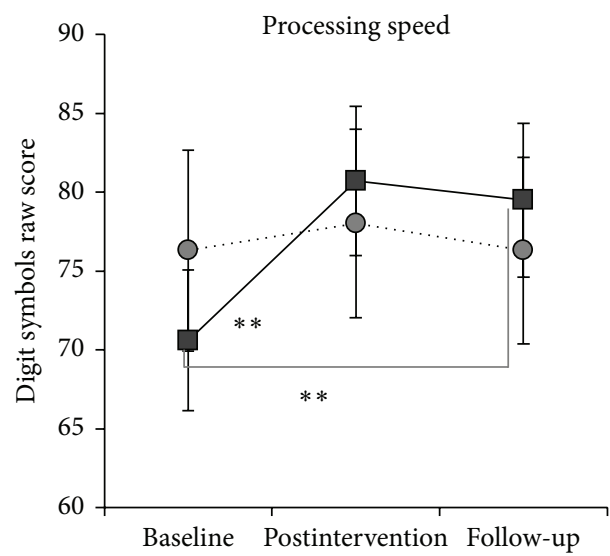

(c)

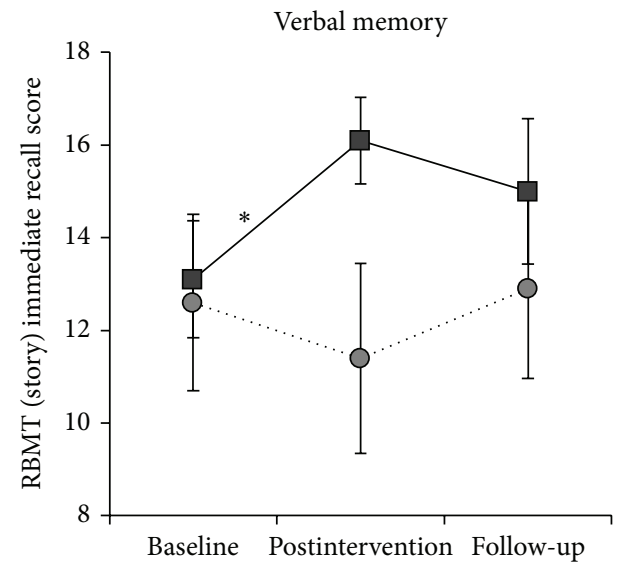

(b)

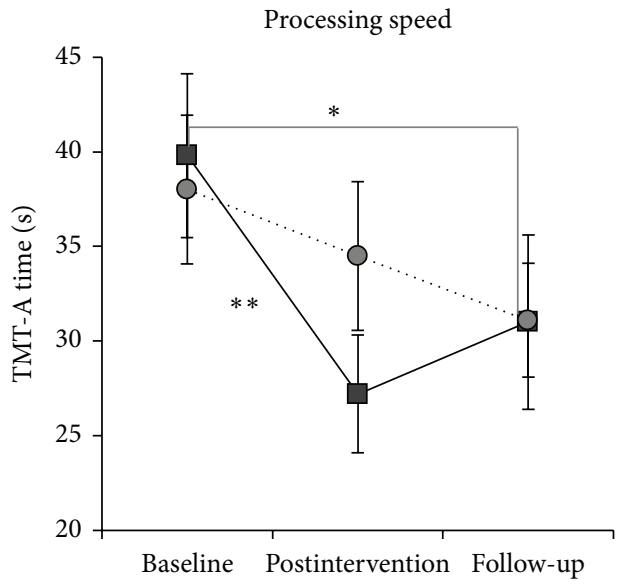

(d)

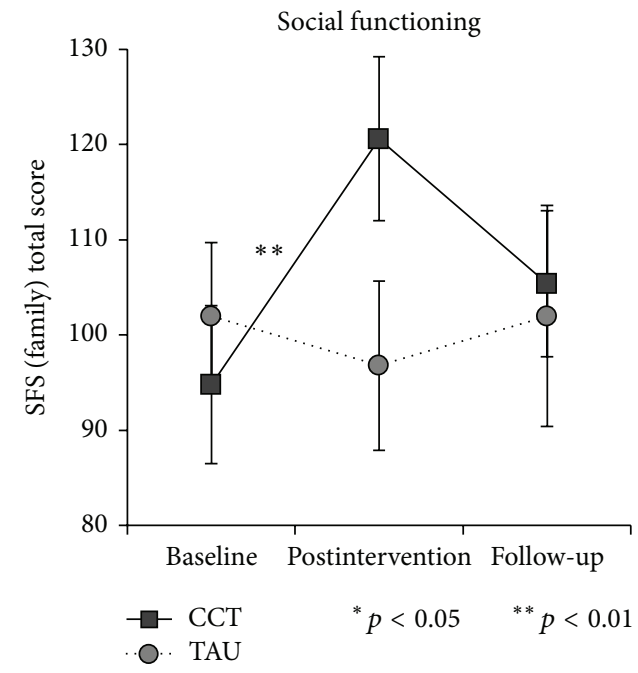

(e)

Figure 2: Means and standard deviations by evaluation times and outcome scores for each group. Square: compensatory cognitive training group; Circle: treatment as usual group. ((a), (b)) For verbal memory, ((c), (d)) for processing speed, and (e) for social functioning. The horizontal axis indicates evaluation times. Baseline: before CCT intervention, postintervention: after 3-month CCT intervention, and followup: 3 months after postintervention. The vertical axis indicates outcome scores. (a) Total score (0-48) of the Japanese Verbal Learning Test, (b) immediate recall score (0-25) of the story subtest from the Rivermead Behavioural Memory Test, (c) raw score (0-133) of the digit symbol subtest from the Wechsler Adult Intelligence Scale-Third Edition, (d) time required to finish the Trail Making Test part A in seconds, and (e) total score (0-226) of Social Functioning Scale, Japanese version, rated by participants' family members. Error bars denote \pm 1 SD. 
3.2. Effects on Everyday and Social Functioning. Of two measures, only the interaction on SFS-J score rated by participants' family members was significant $(F=6.91, p=0.008$, and $\eta_{p}{ }^{2}=0.483$ ). A series of paired comparisons revealed that the CCT group showed significant improvements on social functioning at the postintervention period compared to baseline ( $t=3.44, p=0.004$, and $d=0.92$ ) but that the TAU group showed no change $(t=-0.95, p=1.000$, and $d=0.22$ ). At the follow-up period, both groups did not show any significant changes compared to baseline ( $t s \leq 2.07$, $p s \geq 0.250$, and $d \leq 0.40$ ), suggesting that the improvement effects seen in the CCT group on the SFS-J for families at postintervention were not maintained for several months.

3.3. Effects on Clinical Symptoms. As for clinical symptoms, no significant interactions were observed for both positive and negative symptoms, as seen in Table 3 ( $F s \leq 1.15$, $p s \geq$ 0.341 , and $\eta_{p}^{2} \leq 0.126$ ).

3.4. Effect Sizes. The effect sizes of the CCT intervention were calculated using the change scores from baseline for all measures to contrast the magnitude of the improvement effects between the groups. Table 4 presents the effect sizes of the CCT intervention. Regarding verbal memory, CCT had medium-to-large effect sizes on JVLT immediate recall, delayed recall $(d=0.95,0.69)$, and RBMT story immediate recall and delayed recall $(d=1.00,0.88)$ at postintervention. Furthermore, the equivalent effect sizes of CCT were maintained at follow-up (JVLT, $d=0.55,0.75$; RBMT, $d=$ $0.50,0.85$, resp.).

Large effect sizes of CCT were found in processing speed in both WAIS-III digit symbol $(d=0.97)$ and TMT-A $(d=$ $1.22)$ at postintervention. The equivalent effect size of CCT on digit symbol was maintained at follow-up $(d=1.29)$, but not the effect size on TMT-A $(d=0.19)$. Regarding executive functions, there were small-to-large effect sizes on WCST $(d=0.58)$ and TMT-B $(d=0.33)$ at postintervention. The effect sizes on WCST were maintained at follow-up $(d=$ $0.71)$. On the other hand, the effect size on TMT-B ( $d=$ -0.01 ) was not maintained.

With regard to functional measures, the CCT intervention had medium-to-large effect sizes on the SFS-J rated by the participants themselves and their families at postintervention ( $d=0.47$ and 1.56 , resp.). The effect size of the self-rating was not maintained $(d=0.011)$ while that of family rating decreased $(d=0.049)$. Regarding the measures on clinical symptoms, a small-to-medium effect $(d=0.52)$ was found on positive symptoms at postintervention, and the effect was maintained at follow-up $(d=0.48)$; however, there were no effects on negative symptoms at both times $(d=0.02$ and 0.08 , resp.).

\section{Discussion}

The study examined the effectiveness and applicability of CCT for Japanese patients with schizophrenia. Compared to participants receiving treatment consisting mainly of medication as usual (i.e., the TAU group), those receiving
TABLE 4: Effect sizes for group differences in change scores.

\begin{tabular}{lcc}
\hline & $\begin{array}{c}\text { Postintervention } \\
\text { T2-T1 }\end{array}$ & $\begin{array}{c}\text { Follow-up } \\
\text { T3-T1 }\end{array}$ \\
\hline $\begin{array}{l}\text { Neuropsychological scores } \\
\text { Verbal memory }\end{array}$ & \\
$\quad$ JVLT recall total & 0.95 & 0.55 \\
RBMT immediate recall & 1.00 & 0.50 \\
JVLT delayed recall & 0.69 & 0.75 \\
RBMT delayed recall & 0.88 & 0.85 \\
Processing speed & & \\
$\quad$ Digit symbols raw score & 0.97 & 1.29 \\
$\quad$ TMT-A time & 1.22 & 0.19 \\
Executive functioning & & \\
$\quad$ WCST completed categories & 0.58 & 0.71 \\
$\quad$ TMT-B time & 0.33 & -0.01 \\
Functional outcomes & & \\
Social functioning (SFS-J) & 0.47 & 0.11 \\
(SFS-J for family) & 1.56 & 0.49 \\
Clinical symptoms & & 0.48 \\
SAPS positive symptoms & 0.52 & 0.08 \\
SANS negative symptoms & 0.02 & \\
\hline
\end{tabular}

$\mathrm{T} 1=$ baseline evaluation time, $\mathrm{T} 2=$ postintervention evaluation time, $\mathrm{T} 3=$ follow-up evaluation time, JVLT $=$ Japanese Verbal Learning Test, RBMT = Rivermead Behavioral Memory Test, TMT-A = Trail Making Test-A, WCST = Wisconsin Card Sorting Test, TMT-B $=$ Trail Making Test-B, SFS-J $=$ Social Functioning Scale, Japanese version, SAPS $=$ Scale for Positive Symptoms, and SANS $=$ Scale for Negative Symptoms .

the CCT intervention along with treatment as usual (i.e., the CCT group) showed greater improvements on measures of cognitive functions such as verbal memory, or processing speed, and social functioning. Our results demonstrate that CCT benefits Japanese individuals with schizophrenia. In addition, the high attendance and level of satisfaction rated by the participants in the CCT group indirectly support the notion that this intervention could be effectively applied on Japanese individuals with schizophrenia. The high attendance rate in the CCT group could also imply that they took advantage of the strategies for prospective memory such as the use of calendar for scheduling. They are expected to acquire this new habit through homework exercises. The participants in the CCT group who completed the intervention $(n=11)$ attended $84.9 \%$ of the sessions. Although this percentage decreased to $78.9 \%$ after considering the two participants who dropped out, overall attendance was still high. Participants also reported that the sessions were well conducted (mean rating $=76.6$ on a $0-100$ visual analogue scale). Although definite judgment on whether some of the homework assignments were completed or not was difficult, all participants executed an assignment to call a therapist and leave a message on Saturday using their calendar, once or more in two sessions, for example. Few participants missed all of their homework assignments at each session.

Since impairments on verbal memory in individuals with schizophrenia have been demonstrated to be particularly severe relative to other cognitive domains $[9,10,57]$, it is 
one of the main targets of CCT. Therefore, the successful replication of improvements on verbal memory performance observed in a previous study [31] is a clear indicator of the methodological validity and effectiveness of the Japanese version we administered in this study. JVLT has three or more versions of parallel tests, which we administered randomly to each participant at the three time points. Influences of repetition are considered to be modest, as seen in the performance of the TAU group on these measures. The participants learned a number of memory strategies in the verbal memory sessions and practiced choosing adequate strategies depending on the circumstances. For example, the encoding strategies, such as the use of categorization or visual imagery, are useful for remembering a list for shopping, people's names, and important information in each participant's real-world circumstances. These strategies are considered relevant in performing the JVLT.

As for the other cognitive domains targeted in the CCT intervention, the participants in the CCT group showed greater improvements on measures of processing speed such as the WAIS-III digit symbol or TMT-A compared to those in the TAU group. Since the participants in both groups showed trends in improvement on these measures to some degree, it is plausible that these measures were subjected to the effect of repetition. Nevertheless, the significant groupby-time interactions indicate that the effect of the CCT intervention should be detected beyond that of repetition seen in the TAU group. Indeed, participants in the CCT group showed improvements in processing speed or sustained the level of attention necessary to perform these tasks quickly. Task attention strategies, such as paraphrasing and selftalk, were practiced in the letter cancellation tasks and card games with the goal of utilizing these strategies in everyday activities under real-world circumstances. These strategies are considered relevant for performance on both tests of processing speed (the TMT and WAIS-III digit symbol task). The previous studies $[30,31]$ reported inconsistent effects of CCT on processing speed, showing that CCT led to improvement on the attention measure (digit span) but no improvement on the WAIS-III digit symbol task. Further studies are needed to investigate the effects of CCT on processing speed.

On the other hand, the CCT group did not show significant changes on measures of executive function, which is consistent with previous studies [30,31]. The ceiling effects observed in WCST could make it difficult to examine the effectiveness of the intervention on executive functions in this study and possibly in previous studies [30,31]. Careful selection of additional assessment tools, along with some revisions in the CCT program, might be necessary for examining executive function in future studies.

The effect sizes associated with CCT on cognitive measures at postintervention were as follows: medium-to-large (0.69-1.00) on verbal memory, large (0.97-1.22) on processing speed, and small-to-medium (0.33-0.58) on executive function. Additionally, the effect sizes on social functioning were much larger (0.47-1.56). These results suggest that CCT has promising effects on cognitive performance and that improvements in social functioning occur as well when CCT is applied to Japanese individuals with schizophrenia. These effect sizes were somewhat larger than those in a recent report [31] but are generally similar to those in the first report by Twamley et al. [30]. The results of the previous studies $[30,31]$ showed that effect sizes for functional measures such as subjective quality of life and functional capacity were larger than those for cognitive measures. Consistent with these findings, we observed the same tendencies in this study. Conversational attention strategies, including paraphrasing and asking questions, were practiced by discussing daily topics and sample scenarios with other participants in the sessions. Somewhat larger effect sizes seen in the functional measures suggest that these strategies might encourage patients to take part in social interactions in everyday life and that these strategies possibly had an effect on social functioning independent of the cognitive aspects.

Although effect sizes on cognitive measures were smaller than those on functional measures, significant improvement effects of this compensatory approach on cognitive performance, which has been reported previously [30, 31], were replicated in this study. As Twamley et al. [30] pointed, it is difficult to know whether these effects of CCT represent an attenuation of cognitive deficits or an increase in the ability to compensate for them. Nevertheless, we believe it is unlikely that the intervention is correcting underlying cognitive deficits, because the CCT intervention does not use any drills to improve the cognitive capability itself. Similarly, the improvements on neuropsychological tests seen in the CCT group may reflect the utilization of the strategies participants acquired in the CCT, because none of the neuropsychological tests implemented as cognitive measures was referred to in the sessions. This also implies that the cognitive strategies coached can easily be generalized in various situations, as long as the strategies are applicable. For clarification of the mechanism of action in this compensatory intervention, in future studies, we should include a variety of measures on which the compensatory strategies show improvements or no effects.

In light of the aim of cognitive training, it is worth noting that the improvements on cognitive measures such as verbal memory or processing speed were maintained over a three-month follow-up period. Medium-to-large effects on verbal memory and those on measures of processing speed (WAIS-III digit symbol) and executive function (WCST) were maintained. These results are roughly consistent with those in previous studies [30,31]. Although we did not collect data on the frequency and way in which the strategy was used by CCT participants in everyday living, we could expect, from the above, that the participants made the strategies their new habits and generalized them to everyday tasks. Further studies are required to examine the generalization and availability of the strategies in real-world circumstances.

The impressive improvements on social functioning were moderately maintained (0.49) at follow-up. However, the effect size decreased compared to that of the postintervention period. These results suggest that social interactions or instructions encouraging interpersonal behaviors or prosocial activities provided by the CCT intervention contribute to improvements on social functioning immediately after 
CCT. It is reported that the cognitive remediation provided in conjunction with adjunctive psychiatric rehabilitations has significantly stronger effects compared to cognitive remediation alone $[24,25]$. However, in this study, we did not control for psychosocial intervention (excluding the CCT sessions) in keeping with previous work [31]. Conjunction with periodical psychosocial intervention may be effective in maintaining the improvement effects on social functioning for a longer period of time.

Regarding clinical symptoms, although significant group differences were not observed, CCT had small-to-medium effects $(0.47-0.51)$ on positive symptoms. This result raises questions on whether the improvement effects of CCT on cognitive performance are associated with symptomatology. Therefore, we also conducted correlational analyses on positive symptoms and cognitive function measures. The results showed that positive symptoms were negatively correlated with WCST $(r=-0.91, p=0.000)$, but not with any other cognitive measures ( $r s \leq|0.38|, p \geq 0.070$ ), suggesting that the observed effects of CCT on measures of verbal memory and processing speed have little association with the severity of positive symptoms. Nevertheless, significant correlations between positive symptoms and executive functions represent the possibility that a certain type of cognition is not independent of positive symptoms. The relationship between clinical symptoms and cognitive abilities should be examined in future studies in order to investigate the effectiveness of CCT on cognitive functions more optimally. On the other hand, the previous study [30] showed significant effects on negative symptoms but not positive symptoms. This issue should be carefully examined using larger sample sizes.

Although our results are potentially fruitful, this pilot study has several limitations. The small sample size limits the generalizability of our findings. Regarding the study protocol, we did not employ a rigorous randomization procedure, assessment of treatment fidelity, active control group, or group allocation masking. Although the previous metaanalyses showed that variation in methodological quality (such as lacking these procedures) in cognitive remediation intervention studies had no effect on most cognitive and functioning outcomes [24], these limitations would need to be addressed in future studies. In our results, the effect size of social functioning rated by the participants' families was larger than those rated by participants themselves, suggesting that the expectation of the participants' families biased their evaluations. The result of larger effects in functional outcomes than for cognitive functions is consistent with previous studies [30, 31]. However, it is possible that nonspecific factors including social contact in weekly group sessions could enhance arousal and affect social functioning as well as some of the cognitive measures, in which the effects were attenuated during the follow-up period. Additionally, we could not exclude the interpretation that the CCT participants might be motivated to put more effort into the neuropsychological tests at postintervention and follow-up than that by the TAU participants, with expectation for positive effects of the CCT intervention. In order to clarify these issues, a study of CCT using an active control group to control therapist-time and attention is needed. Finally, Twamley et al. [31] reported a high rate of dropout, but only two participants dropped out during the CCT intervention in our study. To enhance the applicability of this approach, strategies for maintaining participation in CCT sessions are worth investigating in the future.

In conclusion, this pilot study provides the first piece of preliminary evidence on the effectiveness and applicability of CCT for Japanese individuals with schizophrenia. The results suggest that the CCT intervention substantially benefits not only cognitive performance but also functional outcomes in this population. The results of the previous studies were generally replicated, ensuring that the advantages of CCT were not lost in the Japanese version. On the other hand, some different patterns of results were observed in this study, which provides us with new insights relevant to CCT itself and to issues specific to the Japanese version. The promising results observed in this pilot study would encourage further research on CCT in Japan. Investigation into the biological basis of the effects of cognitive remediation is a key challenge. As several studies using cognitive remediation programs based on restorative approaches have shown that cognitive remediation has positive effects on brain structure, brain function, and biomarkers such as brain-derived neurotrophic factors [58-60], studies on CCT are expected to lead to further investigations into its effectiveness from multiple viewpoints, including its neural basis or functional mechanism.

\section{Conflict of Interests}

The authors declare that there is no conflict of interests regarding the publication of this paper.

\section{Acknowledgments}

This study was supported by a Grant-in-Aid for Scientific Research (B) 20330141, 26285155 and Grant-in-Aid for Scientific Research on Innovative Areas 26118707 from the Japan Society for the Promotion of Science (JSPS). The authors thank Dr. Twamley for providing them with the CCT manual and allowing them to use it.

\section{References}

[1] J. M. Gold, "Cognitive deficits as treatment targets in schizophrenia," Schizophrenia Research, vol. 72, no. 1, pp. 21-28, 2004.

[2] A.-K. J. Fett, W. Viechtbauer, M.-D. Dominguez, D. L. Penn, J. van Os, and L. Krabbendam, "The relationship between neurocognition and social cognition with functional outcomes in schizophrenia: a meta-analysis," Neuroscience and Biobehavioral Reviews, vol. 35, no. 3, pp. 573-588, 2011.

[3] M. F. Green, "What are the functional consequences of neurocognitive deficits in schizophrenia?" American Journal of Psychiatry, vol. 153, no. 3, pp. 321-330, 1996.

[4] M. F. Green, R. S. Kern, D. L. Braff, and J. Mintz, "Neurocognitive deficits and functional outcome in schizophrenia: are we measuring the 'right stuff'?" Schizophrenia Bulletin, vol. 26, no. 1, pp. 119-136, 2000. 
[5] M. F. Green, R. S. Kern, and R. K. Heaton, "Longitudinal studies of cognition and functional outcome in schizophrenia: implications for MATRICS," Schizophrenia Research, vol. 72, no. 1, pp. 41-51, 2004.

[6] K. H. Nuechterlein, K. L. Subotnik, M. F. Green et al., "Neurocognitive predictors of work outcome in recent-onset schizophrenia," Schizophrenia Bulletin, vol. 37, supplement 2, pp. S33-S40, 2011.

[7] R. M. Bilder, R. S. Goldman, D. Robinson et al., "Neuropsychology of first-episode schizophrenia: initial characterization and clinical correlates," American Journal of Psychiatry, vol. 157, no. 4, pp. 549-559, 2000.

[8] R. W. Heinrichs and K. K. Zakzanis, "Neurocognitive deficit in schizophrenia: a quantitative review of the evidence," Neuropsychology, vol. 12, no. 3, pp. 426-445, 1998.

[9] R. I. Mesholam-Gately, A. J. Giuliano, K. P. Goff, S. V. Faraone, and L. J. Seidman, "Neurocognition in first-episode schizophrenia: a meta-analytic review," Neuropsychology, vol. 23, no. 3, pp. 315-336, 2009.

[10] M. Matsui, H. Yuuki, K. de Kato et al., "Schizotypal disorder and schizophrenia: a profile analysis of neuropsychological functioning in Japanese patients," Journal of the International Neuropsychological Society, vol. 13, no. 4, pp. 672-682, 2007.

[11] W. J. Brewer, S. M. Francey, S. J. Wood et al., "Memory impairments identified in people at ultra-high risk for psychosis who later develop first-episode psychosis," The American Journal of Psychiatry, vol. 162, no. 1, pp. 71-78, 2005.

[12] R. E. Carrión, T. E. Goldberg, D. McLaughlin, A. M. Auther, C. U. Correll, and B. A. Cornblatt, "Impact of neurocognition on social and role functioning in individuals at clinical high risk for psychosis," American Journal of Psychiatry, vol. 168, no. 8, pp. 806-813, 2011.

[13] P. Jones, B. Rodgers, R. Murray, and M. Marmot, "Child developmental risk factors for adult schizophrenia in the British 1946 birth cohort," The Lancet, vol. 344, no. 8934, pp. 1398-1402, 1994.

[14] T. A. Niendam, C. E. Bearden, I. M. Rosso et al., "A prospective study of childhood neurocognitive functioning in schizophrenic patients and their siblings," The American Journal of Psychiatry, vol. 160, no. 11, pp. 2060-2062, 2003.

[15] A. L. Hoff, C. Svetina, G. Shields, J. Stewart, and L. E. DeLisi, "Ten-year longitudinal study of neuropsychological functioning subsequent to a first episode of schizophrenia," Schizophrenia Research, vol. 78, no. 1, pp. 27-34, 2005.

[16] C.-H. Lin, C.-L. Huang, Y.-C. Chang et al., "Clinical symptoms, mainly negative symptoms, mediate the influence of neurocognition and social cognition on functional outcome of schizophrenia," Schizophrenia Research, vol. 146, no. 1-3, pp. 231-237, 2013.

[17] G. Heydebrand, M. Weiser, J. Rabinowitz, A. L. Hoff, L. E. DeLisi, and J. G. Csernansky, "Correlates of cognitive deficits in first episode schizophrenia," Schizophrenia Research, vol. 68, no. 1, pp. 1-9, 2004.

[18] D. S. O'Leary, M. Flaum, M. L. Kesler, L. A. Flashman, S. Arndt, and N. C. Andreasen, "Cognitive correlates of the negative, disorganized, and psychotic symptom dimensions of schizophrenia," Journal of Neuropsychiatry and Clinical Neurosciences, vol. 12, no. 1, pp. 4-15, 2000.

[19] J. Ventura, G. S. Hellemann, A. D. Thames, V. Koellner, and K. H. Nuechterlein, "Symptoms as mediators of the relationship between neurocognition and functional outcome in schizophrenia: a meta-analysis," Schizophrenia Research, vol. 113, no. 2-3, pp. 189-199, 2009.

[20] J. Ventura, K. L. Subotnik, A. Ered et al., "The relationship of attitudinal beliefs to negative symptoms, neurocognition, and daily functioning in recent-onset schizophrenia," Schizophrenia Bulletin, vol. 40, no. 6, pp. 1308-1318, 2014.

[21] M. Davidson, S. Galderisi, M. Weiser et al., "Cognitive effects of antipsychotic drugs in first-episode schizophrenia and schizophreniform disorder: a randomized, open-label clinical trial (EUFEST)," American Journal of Psychiatry, vol. 166, no. 6, pp. 675-682, 2009.

[22] R. S. E. Keefe, R. M. Bilder, S. M. Davis et al., "Neurocognitive effects of antipsychotic medications in patients with chronic schizophrenia in the CATIE trial," Archives of General Psychiatry, vol. 64, no. 6, pp. 633-647, 2007.

[23] T. E. Goldberg, R. S. Goldman, K. E. Burdick et al., "Cognitive improvement after treatment with second-generation antipsychotic medications in first-episode schizophrenia: is it a practice effect?" Archives of General Psychiatry, vol. 64, no. 10, pp. 1115$1122,2007$.

[24] T. Wykes, V. Huddy, C. Cellard, S. R. McGurk, and P. Czobor, "A meta-analysis of cognitive remediation for schizophrenia: methodology and effect sizes," American Journal of Psychiatry, vol. 168, no. 5, pp. 472-485, 2011.

[25] S. R. McGurk, E. W. Twamley, D. I. Sitzer, G. J. McHugo, and K. T. Mueser, "A meta-analysis of cognitive remediation in schizophrenia," The American Journal of Psychiatry, vol. 164, no. 12, pp. 1791-1802, 2007.

[26] S. Ikezawa, T. Mogami, Y. Hayami et al., "The pilot study of a Neuropsychological Educational Approach to Cognitive Remediation for patients with schizophrenia in Japan," Psychiatry Research, vol. 195, no. 3, pp. 107-110, 2012.

[27] M. Matsui, H. Arai, M. Yonezawa, T. Sumiyoshi, M. Suzuki, and M. Kurachi, "The effects of cognitive rehabilitation on social knowledge in patients with schizophrenia," Applied Neuropsychology, vol. 16, no. 3, pp. 158-164, 2009.

[28] S. Pu, K. Nakagome, T. Yamada et al., "A pilot study on the effects of cognitive remediation on hemodynamic responses in the prefrontal cortices of patients with schizophrenia: a multi-channel near-infrared spectroscopy study," Schizophrenia Research, vol. 153, no. 1-3, pp. 87-95, 2014.

[29] S. Sato, K. Iwata, S.-I. Furukawa, Y. Matsuda, N. Hatsuse, and E. Ikebuchi, "The effects of the combination of cognitive training and supported employment on improving clinical and working outcomes for people with Schizophrenia in Japan," Clinical Practice \& Epidemiology in Mental Health, vol. 10, pp. 18-27, 2014.

[30] E. W. Twamley, G. N. Savla, C. H. Zurhellen, R. K. Heaton, and D. V. Jeste, "Development and pilot testing of a novel compensatory cognitive training intervention for people with psychosis," The American Journal of Psychiatric Rehabilitation, vol. 11, no. 2, pp. 144-163, 2008.

[31] E. W. Twamley, L. Vella, C. Z. Burton, R. K. Heaton, and D. V. Jeste, "Compensatory cognitive training for psychosis: effects in a randomized controlled trial," Journal of Clinical Psychiatry, vol. 73, no. 9, pp. 1212-1219, 2012.

[32] S. Barlati, G. Deste, L. de Peri, C. Ariu, and A. Vita, "Cognitive remediation in schizophrenia: current status and future perspectives," Schizophrenia Research and Treatment, vol. 2013, Article ID 156084, 12 pages, 2013. 
[33] A. Medalia and A. M. Saperstein, "Does cognitive remediation for schizophrenia improve functional outcomes?" Current Opinion in Psychiatry, vol. 26, no. 2, pp. 151-157, 2013.

[34] N. C. Andreasen, The Scale for the Assessment of Positive Symptoms (SAPS), The University of Iowa, Iowa City, Iowa, USA, 1984.

[35] N. C. Andreasen, The Scale for the Assessment of Negative Symptoms (SANS), The University of Iowa, Iowa City, Iowa, USA, 1983.

[36] American Psychiatric Association, Diagnostic and Statistical Manual of Mental Disorders, Text Revision (DSM-4-TR), American Psychiatric Association, Washington, DC, USA, 2000.

[37] K. Matsuoka and Y. Kim, Japanese Adult Reading Test: JART, Shinkou Igaku Shuppan, Tokyo, Japan, 2006.

[38] A. Inagaki and T. Inada, "Dose equivalence of new psychotropic drugs-version 6: risperidone extended-release tablets," Rinsyou Seishin Yakuri, vol. 15, no. 3, pp. 397-404, 2012 (Japanese).

[39] L. Clare, P. J. McKenna, A. M. Mortimer, and A. D. Baddeley, "Memory in schizophrenia: what is impaired and what is preserved?" Neuropsychologia, vol. 31, no. 11, pp. 1224-1241, 1993.

[40] S. Kéri, A. Juhász, Á. Rimanóczy et al., "Habit learning and the genetics of the dopamine D3 receptor: evidence from patients with schizophrenia and healthy controls," Behavioral Neuroscience, vol. 119, no. 3, pp. 687-693, 2005.

[41] P. J. Bayley, J. C. Frascino, and L. R. Squire, "Robust habit learning in the absence of awareness and independent of the medial temporal lobe," Nature, vol. 436, no. 7050, pp. 550-553, 2005.

[42] S. R. McGurk and H. Y. Meltzer, "The role of cognition in vocational functioning in schizophrenia," Schizophrenia Research, vol. 45, no. 3, pp. 175-184, 2000.

[43] W. Spaulding, D. Reed, D. Storzbach, M. Sullivan, M. Weiler, and C. Richardson, "The effects of a remediational approach to cognitive therapy for schizophrenia," in Outcome and Innovation in Psychological Treatment of Schizophrenia, T. Wykes, N. Tarrier, and S. Lewis, Eds., pp. 145-160, John Wiley \& Sons, London, UK, 1998.

[44] M. Matsui, H. Yuuki, K. Kato, and M. Kurachi, "Impairment of memory-organization in patients with schizophrenia or schizotypal disorder," Journal of the International Neuropsychological Society, vol. 12, no. 5, pp. 750-754, 2006.

[45] M. Matsui, T. Sumiyoshi, K. Kato, and M. Kurachi, "Development of alternate forms of Japanese verbal learning test," Seishin Igaku, vol. 49, no. 1, pp. 31-34, 2007 (Japanese).

[46] J. M. Gold, C. Randolph, C. J. Carpenter, T. E. Goldberg, and D. R. Weinberger, "Forms of memory failure in schizophrenia," Journal of Abnormal Psychology, vol. 101, no. 3, pp. 487-494, 1992.

[47] S. Watamori, H. Hara, T. Miyamori, and F. Eto, The Rivermead Behavioral Memory Test (RMBT), Japanese Version, Chiba Test Center, Tokyo, Japan, 2002.

[48] B. A. Wilson, J. Cockburn, and A. Baddeley, The Rivermead Behavioral Memory Test (RMBT), Thames Valley Test Company, Bury St Edmunds, UK, 1986.

[49] K. Fujita, H. Maekawa, H. Dairoku, and K. Yamanaka, Wechsler Adult Intelligence Scale, Nihon Bunka Kagakusha, Tokyo, Japan, 3rd edition, 2006, (Japanese).

[50] D. Wechsler, Wechsler Adult Intelligence Scale-Third Edition (WAIS-III), The Psychological Corporation, San Antonio, Tex, USA, 1997.
[51] R. M. Reitan and D. Wolfson, The Halstead-Reitan Neuropsychological Test Battery, Neuropsychology Press, Tucson, Ariz, USA, 1985.

[52] R. K. Heaton, G. J. Chelune, J. K. Talley, G. G. Kay, and G. Curtiss, Wisconsin Card Sorting Test Manual: Revised and Expanded, Psychological Assessment Resources, Odessa, Tex, USA, 1993.

[53] T. Nemoto, C. Fujii, Y. Miura et al., "Reliability and validity of the Social Functioning Scale Japanese version (SFS-J)," Japanese Bulletin of Social Psychiatry, vol. 17, pp. 188-195, 2008 (Japanese).

[54] M. Birchwood, J. Smith, R. Cochrane, S. Wetton, S. Copestake, and M. Birchwood, "The social functioning scale. The development and validation of a new scale of social adjustment for use in family intervention programmes with schizophrenic patients," The British Journal of Psychiatry, vol. 157, no. 6, pp. 853-859, 1990.

[55] H. E. Nelson, "A modified card sorting test sensitive to frontal lobe defects," Cortex, vol. 12, no. 4, pp. 313-324, 1976.

[56] J. Cohen, Statistical Power Analysis for the Behavioral Sciences, Lawrence Erlbaum Associates, Hillsdale, NJ, USA, 2nd edition, 1988.

[57] A. Aleman, R. Hijman, E. H. F. de Haan, and R. S. Kahn, "Memory impairment in schizophrenia: a meta-analysis," The American Journal of Psychiatry, vol. 156, no. 9, pp. 1358-1366, 1999.

[58] S. M. Eack, G. E. Hogarty, R. Y. Cho et al., "Neuroprotective effects of cognitive enhancement therapy against gray matter loss in early schizophrenia: results from a 2-year randomized controlled trial," Archives of General Psychiatry, vol. 67, no. 7, pp. 674-682, 2010.

[59] S. Vinogradov, M. Fisher, C. Holland, W. Shelly, O. Wolkowitz, and S. H. Mellon, "Is serum brain-derived neurotrophic factor a biomarker for cognitive enhancement in schizophrenia?" Biological Psychiatry, vol. 66, no. 6, pp. 549-553, 2009.

[60] B. E. Wexler, M. Anderson, R. K. Fulbright, and J. C. Gore, "Preliminary evidence of improved verbal working memory performance and normalization of task-related frontal lobe activation in schizophrenia following cognitive exercises," American Journal of Psychiatry, vol. 157, no. 10, pp. 1694-1697, 2000 . 


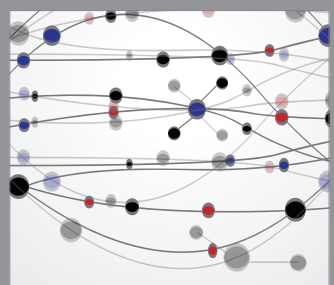

The Scientific World Journal
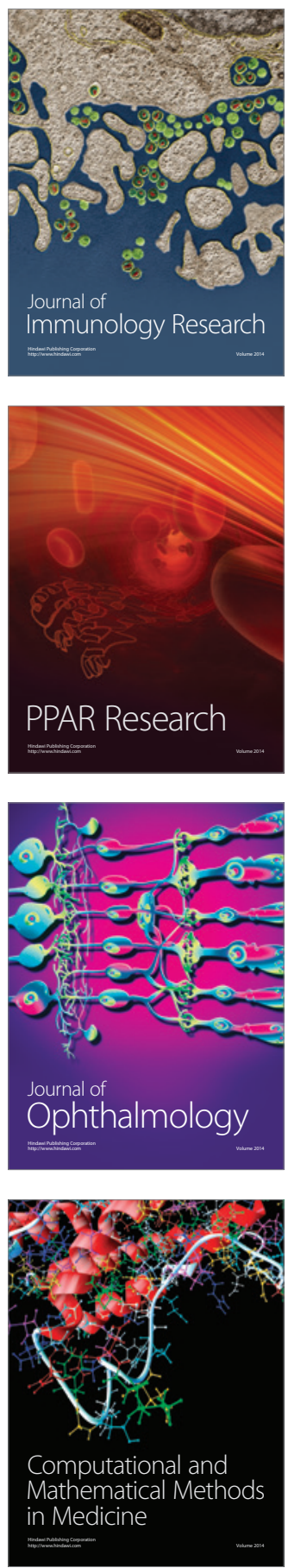

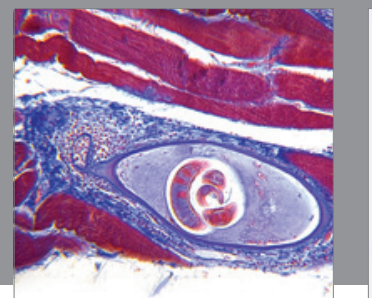

Gastroenterology

Research and Practice
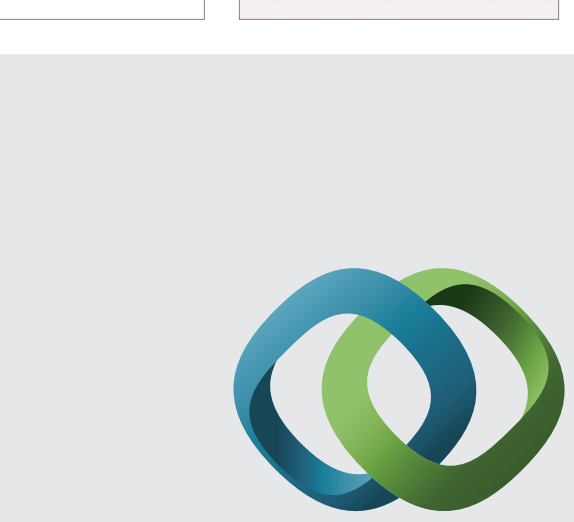

\section{Hindawi}

Submit your manuscripts at

http://www.hindawi.com
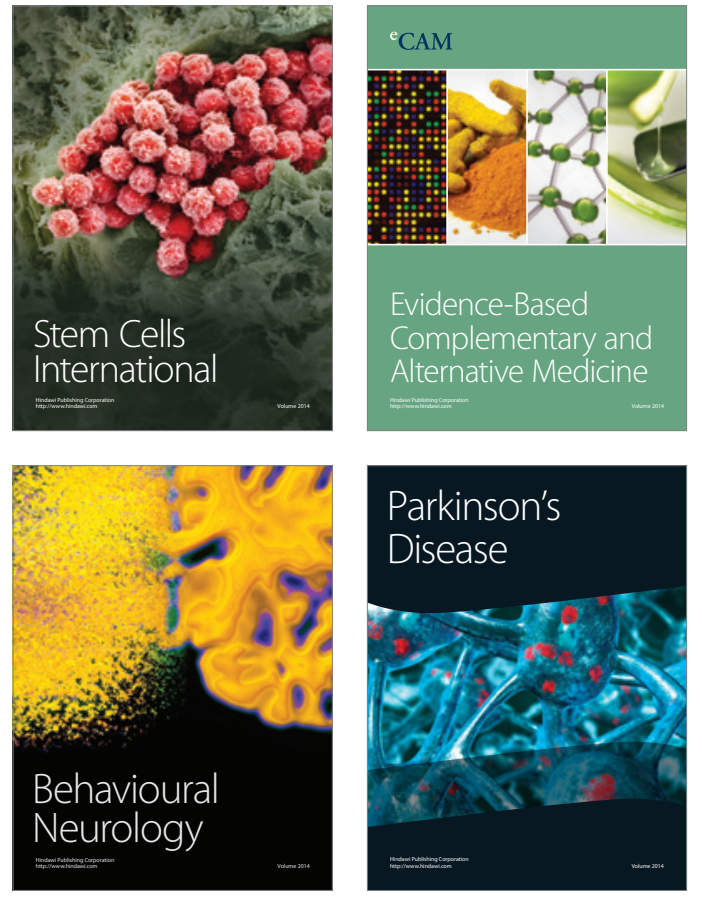
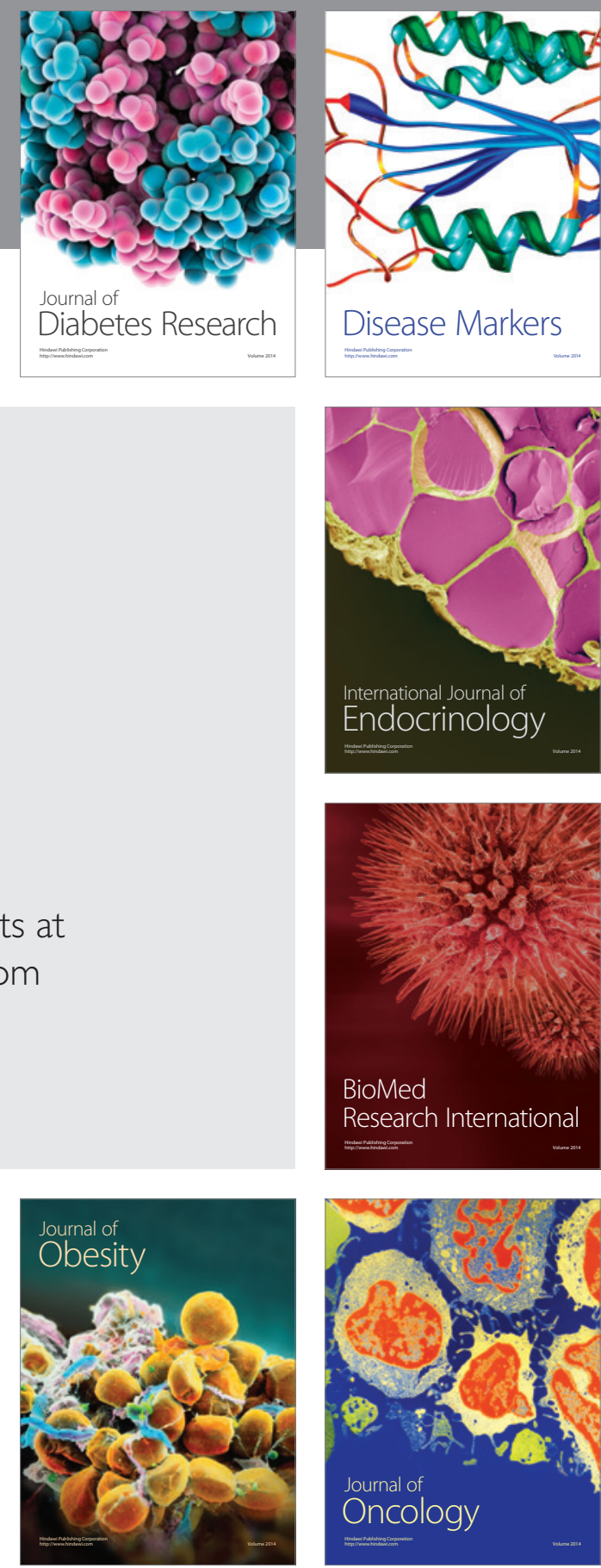

Disease Markers
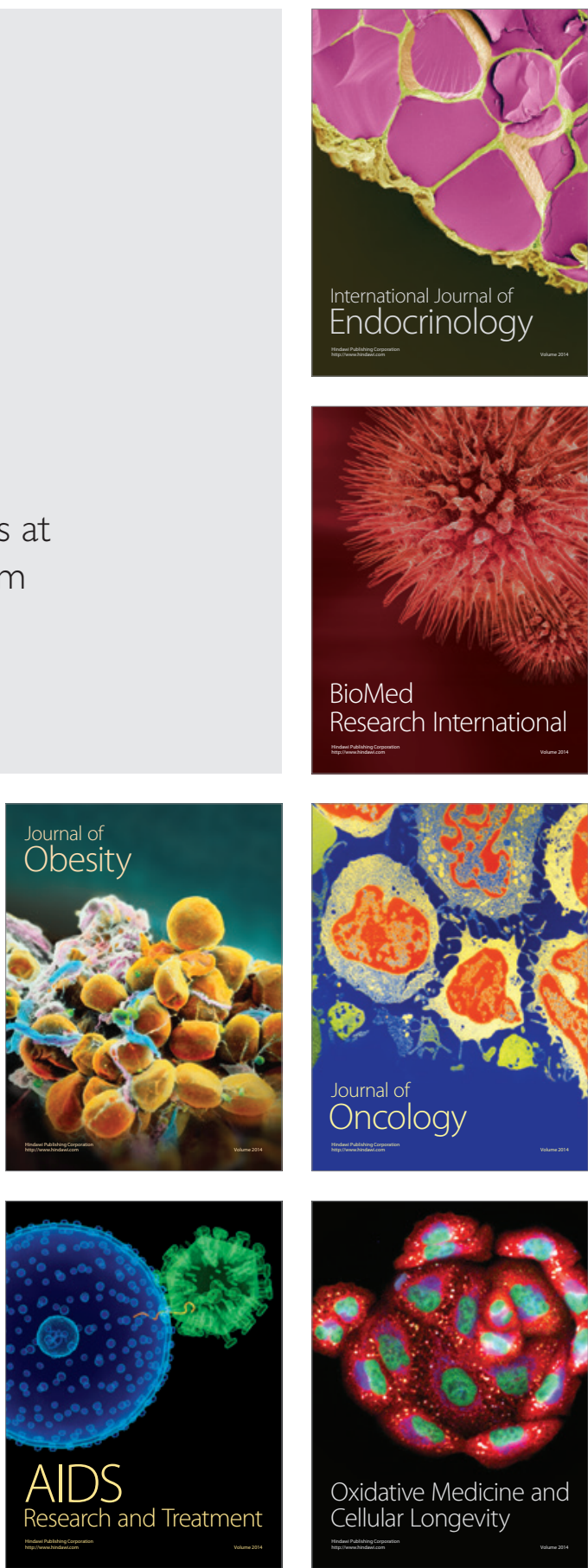